\title{
Research on the distribution automation system based on IEC61850
}

\author{
Xin Hongwang \\ School of Electrical and Electrical Engineering \\ North China Electric Power University \\ Baoding, China \\ E-mail: 67454307@qq.com
}

\author{
Ding Qiaolin \\ School of Electrical and Electrical Engineering \\ North China Electric Power University \\ Baoding, China
}

\begin{abstract}
In order to solve interoperability of the distribution automation devices and Plug and Play issues, propose intelligent power distribution model based on IEC61850, Stratifie according to the method of information: Master level、feeder layer、 the terminal layer、device layer、 layer monitoring. Use the face of the object model of the distribution automation IED, at the same time, use abstract communication service independent of the specific communication technology model ACSI, Establish distribution network automation system communication system Follow the IEC61850 standard.
\end{abstract}

Keywords- IEC61850; Distribution Automation; Modeling; Interoperability;

\section{INTRODUCTION}

A long time, the coverage of China's distribution network automation and practical level of investment of time is insufficient because of the constraints of the level of automation and communication, which results in a lot of blind spots in information monitoring in the distribution network ${ }^{[1,2]}$.

For the above, the use of modern high-speed computer and communications technology, real-time operation of the grid, the grid structure, equipment, user and geographic graphic information integration constitute the complete automation system to achieve operation monitoring, management and information system in the distribution network automation ${ }^{[3]}$.

\section{DISTRIBUTION NETWORK AUTOMATION}

Conventional distribution network ${ }^{[4]}$ has the following shortcomings: Communication only between the terminal and the main station or the sub station; don't exchange data between the distribution terminal, don't achieve local control functions; Because data is not transparent transmission between the terminal and the master, configuration management and maintenance are heavy workload; it is difficult to transfer the fault recorder, power quality disturbance records batch data because of limited channel bandwidth; it is difficult for devices from different manufacturers to inter-plug Internet; Terminal device can not plug and play immediately; Need communication configuration in the side of the station, on both sides of the master, likely to cause error in data dislocation, the data type mismatch.

IEC61850 provides a unified standard for substation automation to achieve interoperability between different smart devices, Reference to IEC61850 techniques and methods, introduce it into the distribution network automation and establish distribution automation communication system followed the IEC61850 standard in order to achieve the object model of terminal equipment and the standardization of information exchange model; Take advantage of the substation Description Language (SCL) ${ }^{[5]}$ to achieve self-description of the terminal equipment, achieve distribution automation equipment to plug and play immediately, and solve the problem of many equipment automation access in the distribution network.

\section{Distribution AUtOMATION INFORMATION MODEL}

Logically, the distribution automation system is divided into five layers according to information stratification method, the layer of the master, feeder (ring) layer, the terminal layer (FTU, of TTU, etc.), the device layer (switches, current transformers (TA), the voltage transformer (TV)), and listen layer. Show in Figure 1.

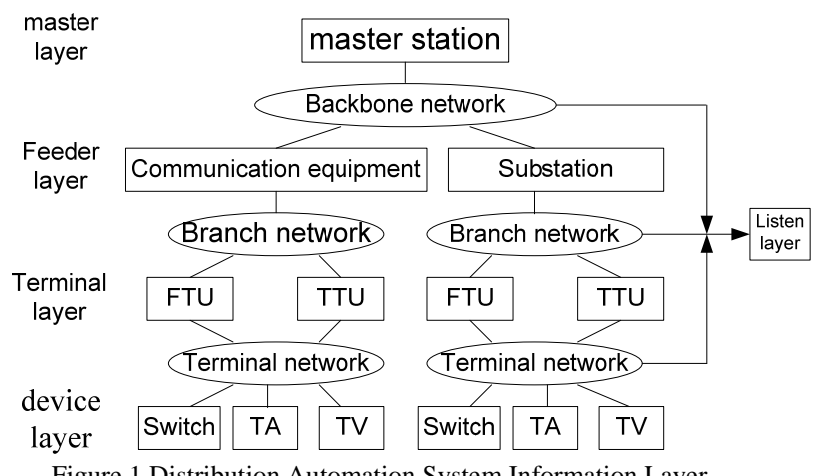

Figure 1 Distribution Automation System Information Layer

Master layer in the control center is the heart and brain of distribution automation; Feeder layer for monitoring a pressure feeder lines includes network equipment in the substation and intelligent electronic devices of the entire feeder; Terminal layer is located on a specific line of Intelligent Electronic Device (IED) [6], including FTU, FTU, TTU, and DTU. The device layer is the implementation of the smart device layer, including the isolation switch, current transformer (TA), the voltage transformer (TV); Listen layer is composed by the monitoring equipment and recording devices, which is primarily responsible for the recording and storage of all network traffic packets to provide effective adjunct for troubleshooting: 


\section{A. Master layer}

Master layer in the control center monitor and control the whole distribution network, isolation and power restoration, fault location, which is the core of the entire distribution network monitoring and management system. Master layer through the front-end data achieve access and management of a large number of IDE.

In the IEC61850 distribution network communication system, the pre-communications processor and on-site terminal communication devices communicate on the data pre-processing to reduce the burden on the server, in addition to the system clock synchronization, channel monitoring and switching to other automation systems or MIS system forwarded the data and other functions. Front-end up connect to the master LAN, with back-machine exchange of data; Down to client / server model with a variety of on-site terminal device communication, collection distribution network monitor real-time operating data, and issue a regulation command.

\section{B. Feeder layer}

The feeder layer located in the substation consists of a feeder or a ring online distribution terminal, Feeder layer achieve logical processing functions of a feeder by mutual cooperation between these distribution terminals. Feeder layer devices as agent communication between the distribution master and terminals Feeder layer device play role to optimize information transmission and facilitate communication system network. The distribution electronic stations are usually placed in the substation or large switching station responsible for the distribution terminal in the area data set forwarding.

\section{Terminal layer}

Terminal layer is located in the automation field devices on the distribution network line, including FTU, TTU, and FTU. Distribution terminal to complete the following functions the word "data" is plural, not singular.

- Short-circuit fault detection functions

- Harmonic measurement functions

- Small current grounding fault detection functions.

- Intelligent charging management

- $\quad$ Billing system electronic segment billing

- $\quad$ SCADA measurement and control functions.

D. device layer

The device layer includes voltage, current transformers, circuit breakers, isolating switches and transformer primary equipment:

- Real-time electrical detection (such as voltage and current signals)

- Running status of the device parameters to detect

- Operational control (such as the circuit breaker closing operation)

\section{E. Listen layer}

Listen layer is composed by the monitoring equipment and recording devices, which is mainly responsible for the recording and storage of all network traffic packets, effective adjunct for the operation and maintenance personnel to troubleshoot.

The monitoring equipment design principles are: monitoring equipment in the case of the normal, failure and loss of power can not affect the normal communication of the network being monitored, and can not interfere with listening communication network signal.

Recording equipment in the substation environment is responsible for noncontiguous and complete storage network communication packets. IEC61850 monitoring and control associated artesian and protection of the fast packet and electronic current, voltage transformer measurement packets pass in the link layer, network communication protocols must be recorded by the recording equipment including communication protocol link layer and above all, monitor traffic to determine the log file is saved time and record storage media capacity, when the log file reaches the set capacity, take the way of covering the history record.

\section{Distribution Automation IED AND Modeling}

Current modeling of IEC61850 substation automation equipment has been developed to be used in different occasions, the intelligent power distribution terminal, including FTU, RTU, TTU, and DTU. Show in Figure 2.

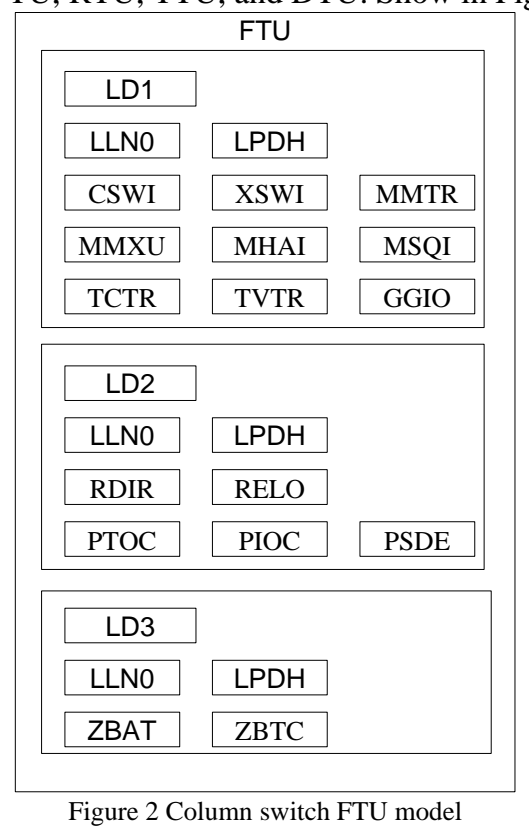

Each distribution terminal device contains three LD (logical device) The LD1 complete SCADA functions, and realization of remote communication, telemetry, remote control and remote adjustment; LD2, complete fault detection and protection; LD3 complete intelligent power management

Each logical device includes the LLN0, and LPDH node, LLN0 contains the physical device IED control IED self-test, LPDH serve as the public information model of the physical device IED, such as the nameplate information, the device self-test results. 
For some special features such as distribution automation, small current grounding protection, fault location, charge management and super capacitor, information modeling requires a combination of the characteristics of distribution networks and distribution lines.

\section{A. LD1}

LD1 is the monitoring and control function modules, including the switching controller (CSWI), load switch (XSWI), phase sequence and unbalanced (MSQI) measurement (MMXU), current transformer (TCTR,) voltage transformer (TVTR,), phase do not unrelated to the harmonics and measurement (MMTR), the universal Process I / O (GGIO) and other logical nodes.

The CSWI collects column switch CSWI location status, and column switch with circuit breakers use with breaker (XCBR,) instead of Switches (XSWI)

MHAI logical node is used to calculate the harmonics in the single-phase system. Single-phase system that is a single loop, relative to gender relations. Specific examples harmonic (including harmonics and integer harmonics) instance, may also be instances of inter-wave, depending on the basic value, the frequency $\mathrm{f}$, analysis window.

MMXU acquisition the current and voltage information of distribution lines through TCTR, TVTR, GGIO as generic process I / O logic node can model for inconvenient analog or state, such as soft plate information. MSQI is used to measure the imbalance current. The measurement (MMTR) the electrical energy is used in the calculation of three-phase system, applicable to the billing.

\section{B. $L D 2$}

LD2 for a protection, fault detection module includes instantaneous over current (PIOC) time over current (PTOC), direction components (RDIR,), the fault location (RELO), sensitive to the direction of ground fault (PSDE) logical node.

\section{LD3}

LD3 for intelligent power management module, there are battery (ZBAT) and battery charging management (ZBTC is) and other logical nodes in it Some Common Mistakes.

\section{INFORMATION EXCHANGE (ACSI)}

IEC61850 abstract communication service model ACSI is independent of specific communication technologies, it does not specify communication services which depend on communication media, protocol stacks, as well as specific message encoding format. Defined 14 model, 62 kinds of services in the abstract communication service model ACSI, which basically covers the demand for distribution automation functions

Services in the abstract communication service interface have two sets of basic communication mode. A group uses lent / Service mode, it is mainly used for directory inquiries, read and write data, and control services, and its advantage is the safety of the service, high reliability, the drawback is that real-time is not enough; Another group of Publisher / the Subscriber mode for the GOOSE message is sent, the sampling value, and other services, its advantages are suitable for large volumes of data real-time requirements of the occasion.

\section{SPECIFIC COMmUnication SERVICE Mapping} (SCSM)

Accordance with the IEC61850 standard, information exchange service of the distribution terminal is mapped to the specific means of communication:

- Client / Service mode is mapped to MMS (manufacturing mesh the sage specification) or IEC60870-5-101 to achieve

- Transmission of sampled values reference to IEC 61850-8-1 is mapped

- Time is mapped by simultaneous sampling of the Simple Network Time Protocol

- GOOSE message transmission service is applied to the presentation layer, mapped directly to the ISO/IEC8802-3 Ethernet data link layer according to IEC 61850-8-1 after the abstract syntax of ASN.1 encoding, not via the network and transport layers, and switched Ethernet technology to ensure priority of the transmission of important information, thus ensure real-time packet transmission

\section{CONFIGURATION LANGUAGE}

IEC61850 substation configuration description Language (SCL) is used to describe not only the product information model, communication model but also describes the substation connection between the logical communication connection and between the product relationship, the corresponding relations of the model and field data.

\section{MODEL VALIDATION}

TMW 61850 Test Harness can create and edit SCL which follows the rules of XML syntax, is Microsoft Window applications that support IEC 61850 client test. And TMW 61850 Test Harness's internal package supports IEC 61850 data underlying communication protocol. So that it can be directly used to verify the correctness of the model.

TMW 61850 Test Harness can simulate the simulation distribution terminal following function: (1) custom switch set point changes; (2) remote feedback sent to the remote signaling modification, telemetry changes send alarm events are sent; (3) analog send and receive MMS messages, analog protection event.

Run TMW 61850 Test Harness software on the PC, articulated in the distribution network, comprehensive functional testing of the client system instead of the power distribution terminal. Model validation example is showed in Figure3. 


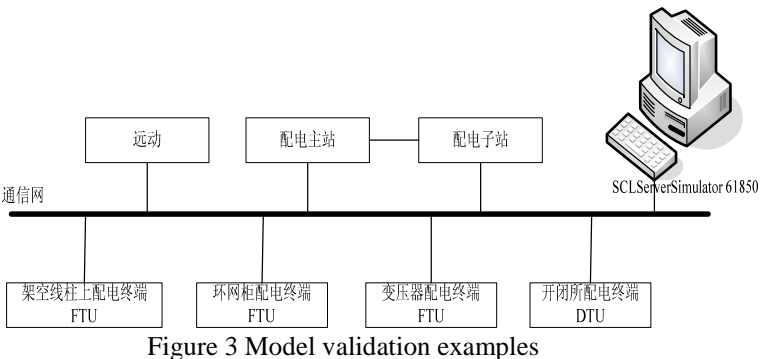

TMW 61850 Test Harness either cans analog monitoring or control functions that can simulate the protective function. Therefore, the software can be tested background monitoring system, remote device station following security engineers, the features and configuration of the electronic distribution station is normal. Through simulation testing, you can ensure that the correct function and configuration of the client, enabling seamless communication of the actual distribution terminal client system. Verification of the client to obtain the information model is showed in Figure4.

\begin{tabular}{|c|c|}
\hline TSHQ cid & $\Leftrightarrow$ Client: TSHQ [10,10.24 105] \\
\hline Hame & Hame \\
\hline \pm Header & $\exists$ TSHQBWIY \\
\hline \pm Communi cation & + GGI01 \\
\hline$\exists$ TSHQ & † LURO \\
\hline$\exists$ accessPoint1 & + LPHD1 \\
\hline$\exists$ Server & $\pm \mathrm{ZBAT} 1$ \\
\hline$\pm \mathrm{XDC} 1$ & $\exists$ TSHQJFUnit \\
\hline$Ð \mathrm{XDC} 2$ & t GGIO1 \\
\hline $\pm X D C 3$ & \\
\hline$\pm \mathrm{XDC} 4$ & \pm LLसO \\
\hline$Ð$ YD1 & \pm LPH1 \\
\hline \pm YD2 & \pm MMXV1 \\
\hline$\exists$ BWIY & \pm TSHQXDC1 \\
\hline$\oplus L \mathrm{~L} M 0$ & \pm TSHQXDC2 \\
\hline \pm ZBAT1 & \pm TSHQXDC3 \\
\hline \pm GGI01 & \pm TSHQXDC4 \\
\hline Ð LPW1 & $\oplus$ TSHQYD1 \\
\hline$\exists$ JFUnit & \pm TSHQYD2 \\
\hline † Lلس & \pm TSHQYDDY \\
\hline \pm MMXV1 & \\
\hline \pm LPHD1 & \\
\hline \pm GGI01 & \\
\hline$Ð$ YDDY & \\
\hline
\end{tabular}

Figure 4 Verification of the client to obtain the information model

\section{CONCLUSION}

In this paper, IEC61850 is introduced to distribution automation, distribution automation system is divided into five levels according to the information layered approach, Use modeling method of object-oriented for distribution automation IED modeling and independent specific communication technology Abstract communication service model ACSI, build distribution automation communication system follow the IEC61850 standard in order to achieve plug-and-play and interoperability of different manufacturers equipment.

\section{REFERENCES}

[1] IEC IEC61850 communication networks and system in substation[s],2004

[2] Mohagheghi Salman, Mousavi Mirrasoul J , Stoupis James, "Modeling distribution automation system components using IEC 61850” , 2009 IEEE Power and Energy Society General Meeting,PES09, July 26, 2009-July 30, 2009, Calgary, AB, Canada, 2009

[3] Qin Lijun, Liu Lin, Shi Junfeng, Distribution automation system according to IEC61850 [ J ] ,Electrical equipment, 2007, 8 (12) : 13-15

[4] Xu Bingyin Li Tianyou. A number of issues of distribution automation. Power system automation $[\mathrm{J}], 2010,34$ (9) : 8186..

[5] Michael Blaha, James Rumbaugh. UML Object modeling and design by the face.Li Haoyang, Yang mei. Beijing : People Post Press, 2006. .

[6] Luo Sibei, Huangrunchang, et al. IED modeling based on objectoriented technology of IEC61850 standard $[\mathrm{J}]$.Automation of Electric Power Systems, 2009, 37 (17) : 88-92.M. Young, The Technical Writer's Handbook. Mill Valley, CA: University Science, 1989. 